\title{
Fate of Nanoparticles in Biological Systems - Quantum Dot Behavior in Bacterial Cells and Biofilms
}

\author{
R. David Holbrook, * J.B. Morrow**, T.K. Maugel*** \\ * Surface and Microanalysis Science Division, National Institute of Standards and \\ Technology, Gaithersburg, MD 20901 \\ ** Biotechnology Division, NIST, Gaithersburg, MD 20901 \\ *** Laboratory for Biological Ultrastructure, University of Maryland, College Park, MD \\ 20742
}

Quantum dots (QDs) are colloidal semiconductor nanocrystals that have been utilized as for fluorescent imaging of bacterial systems [1] and biofilms [2]. Biofilms are communities of microorganisms attached to surfaces that predominate in water/surface interfaces common to nearly all ecosystems [3]. The study of microorganisms at the aqueous/substratum interface is of great interest because bacterial attachment to surfaces is often associated with surface deterioration and corrosion. Extracellular polymeric substances anchor bacteria to the substrate [3] and serve as a survival mechanism in diverse environments including water distribution systems [4]. Decontamination of biofilms by dynamic changes in fluid shear resulting in biofilm deformation and detachment may be a viable alternative to chemical disinfection methods, however, detachment mechanisms have yet to be elucidated.

QDs with two different surface chemistries (amine and carboxyl modified) were utilized to measure solute mobility and diffusion in bacterial cells and biofilms as a function of fluid shear. Bulk surface chemistry measurements were used to predict the governing interfacial interactions between QDs and bacterial cells/biofilms upon initial contact. Interaction energies of QDs with bacterial biofilms, governed by interfacial forces include van der Waals, electrostatics and hydrophobicity (including Lewis acid/base contributions), are predicted to be largely attractive for bacterial and drinking water biofilms grown on PVC pipe.

Diffusion coefficients for QDs in single (Pseudomonas aeruginosa PAO1 and MCF-7 breast cancer cells) and native drinking water bacterial biofilms were dependent on the size and chemistry of the quantum dots and, in general, agreed well with predicted attractive interaction energies. However, uptake of QDs by cells was not observed and was attributed to significant aggregation at or near the cell surfaces (Fig. 1). Shear induced biofilm deformation and increased QD penetration was evident by observation with Confocal Laser Scanning Microscopy (CLSM) via gfp labled PAO1 biofilms (Fig. 2 ). Increased penetration into the biofilm was attributed to shear-induced changes in biofilm morphology and not increases in the QDs kinetic energy.

[1] Kloepfer, J.A. et al., App. Environ. Micro. 71 (2005) 2548.

[2] Chalmers, N.I. et al., Focus on Microscopy (2005) Friedrich Schiller University, Jena, Germany.

[3] Costerton, J.W. et al., Ann. Rev. Micro. 49 (1995) 711.

[4] Hall-Stoodley, L. et al., Nat. Rev. Micro. 2 (2004) 95. 


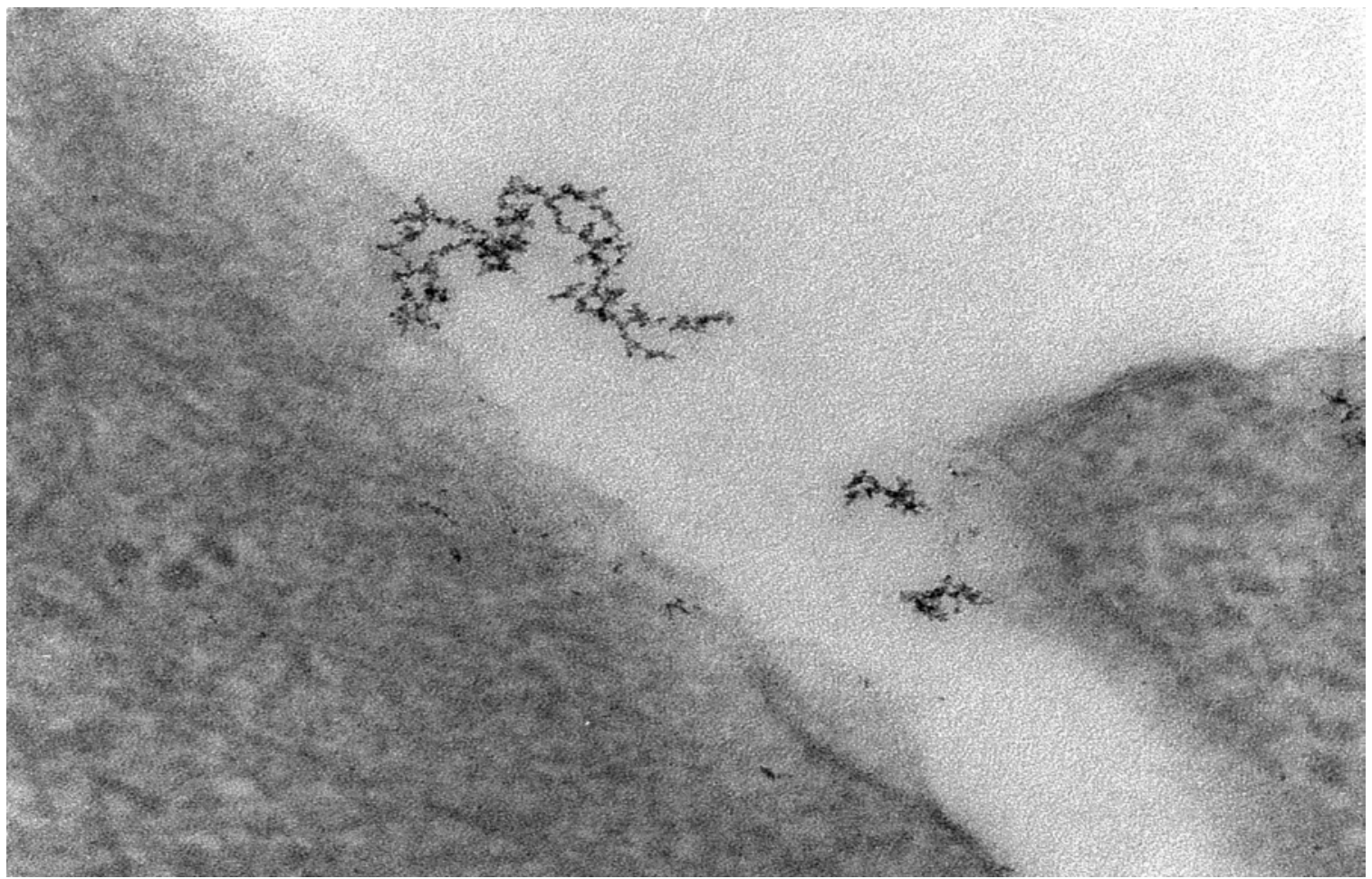

Fig. 1. TEM image of MCF-7 cells and quantum dots $(\mathrm{em}=705 \mathrm{~nm})$. Note chain aggregation of quantum dots. 125,000x.

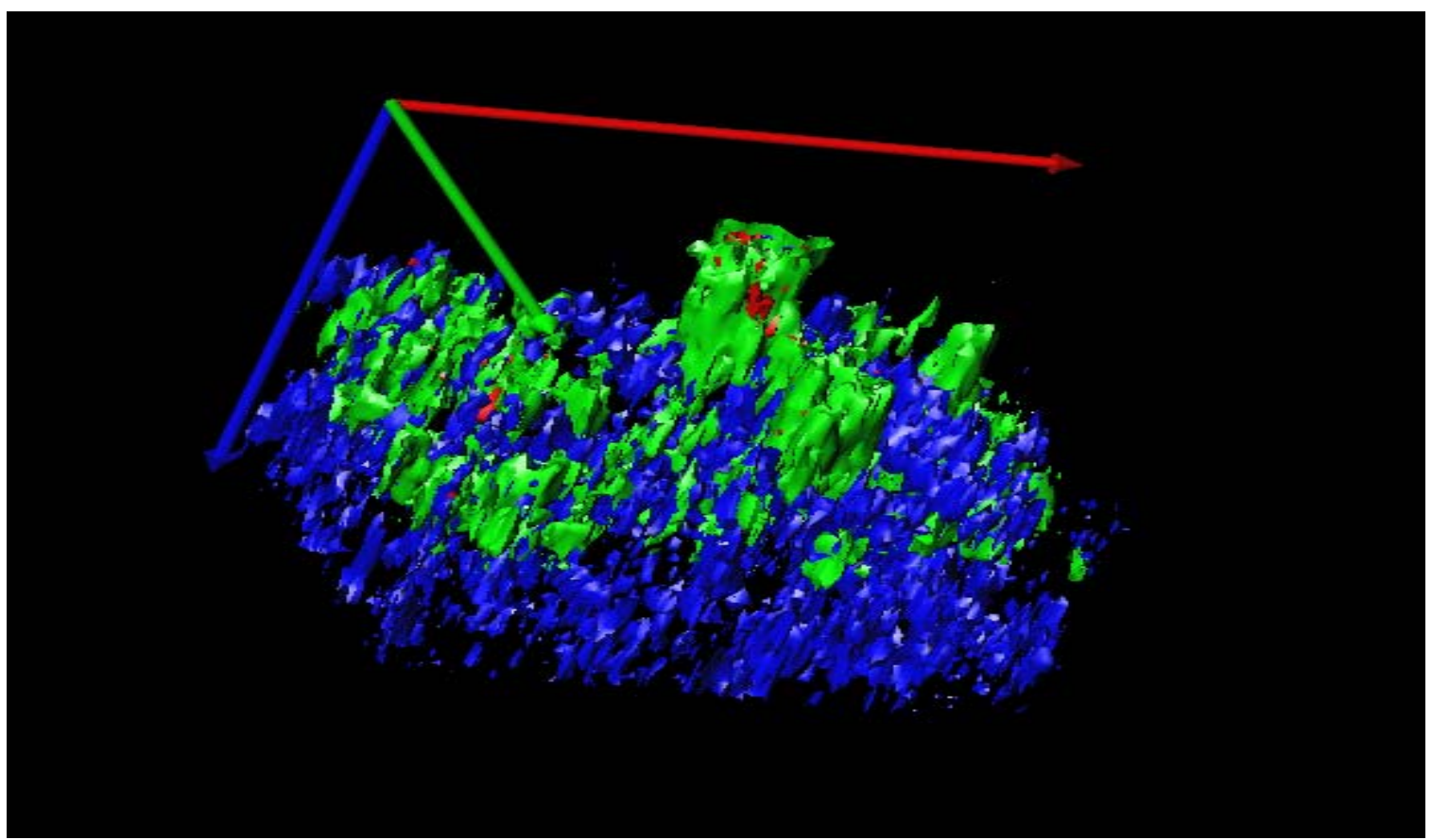

Fig. 2. 3-D reconstruction of laser scanning confocal microscopy image of biofilm sample. Green represents the green fluorescence protein (GFP) of Pseudomonas aeruginosa, red represents carboxylated quantum dots $(\mathrm{em}=655 \mathrm{~nm})$ and blue represents fucose (a polysaccharide common in biofilms). Note that the quantum dots appear to be most closely associated with bacterial surfaces and not fucose. 